\title{
Natural and artificial 'singing' sands
}

Two types of sand that emit audible sounds on being sheared, by wind or other mechanical means, have been reported throughout the world over the past century ${ }^{1-5}$. They are commonly known as squeaking, or singing, and booming sands. The exact mechanism by which sounds from these sands are produced is still unknown. Natural musical sands are thought to have a silica gel-type surface layer on the sand grains. We show here that this layer is crucial for the singing behaviour, as a commercially manufactured silica gel, an artificial sand material, 'sings'.

Many singing sands are well sorted materials with sizes in the range 100-500 $\mu \mathrm{m}$, roughly rounded shapes, and a high content of quartz particles ${ }^{3,6}$, and yet booming sand materials are also found in Kauai, Hawaii, where sands are mostly calcareous in content. We have used Fourier transform infrared (FTIR) spectroscopy to characterize the surface of musical sands ${ }^{7,8}$. One significant feature of the infrared spectrum was a broad band at $3,400 \mathrm{~cm}^{-1}$, extending from $3,700 \mathrm{~cm}^{-1}$ to $2,800 \mathrm{~cm}^{-1}$. This band may be due to clusters of water in an amorphous silica layer on the surface of the sand grains'.

We have previously shown ${ }^{8}$ that sand materials that do not sing can be made to do so by grinding the grains in a mill, periodically removing the fines and renewing the water, leaving a well sorted and surface-polished material. After sufficient grinding, these can achieve the singing or booming state, and they exhibit the characteristic $3,400 \mathrm{~cm}^{-1}$ band.
We have now completed similar studies on the singing sands of Kauai and they too show a $3,400 \mathrm{~cm}^{-1}$ infrared band, indicating that a similar water-silica layer may exist on the surface of these non-quartz sand materials. As the solubility of silica in water is about $1 \mathrm{mM}$ (ref. 10), the formation of such a siliceous layer even on nonquartz sand grains is possible. This similarity in structure between singing sands of vastly different bulk composition led us to hypothesize that this silica gel-type layer may be part of the mechanism of sound generation in these materials. Indeed, the repeated suggestion in the literature that a certain degree of dryness (and thus a specific water content) of these materials is required for such sands to sing fits nicely into the silica gel-type mechanism and the known deliquescent properties of silica gels.

If this layer on the sand grains was the cause of the singing phenomenon, then commercially available silica-gel materials should also 'sing'. We tested this idea using commercial grade silica gel (Fisher Scientific) with a size range of $200-500 \mu \mathrm{m}$. On sharp shaking of a 200-g sample of this material in a glass bottle, a loud sound was emitted, similar to that of natural singing sands. Furthermore, this material gives the characteristic $3,400 \mathrm{~cm}^{-1}$ infrared band and an audio beat pattern in the range of $450 \mathrm{~Hz}$, typical of natural singing sands.

These observations lead us to speculate that, as silica gel is a weakly bonding material (highly hydrated commercial silica gel particles adhere), then silica gel-type layers found in natural sands might allow their particles to stick weakly together. The emission of a coherent wave sound pattern requires a minimal number of such particles to stick together, and such a loose cohesive mass of appropriately sized sand particles moving through the air, either tumbling down a dune or being shaken in a container, could then act as a resonator.

The finding that commercially available silica gel is a 'musical sand' should enable a more detailed study of the phenomenon, and could lead to practical application of these readily available materials in energy converter devices.

Douglas E. Goldsack

Marcel F. Leach

Cindi Kilkenny

Centre in Mining and Mineral Exploration Research, and Department of Physics,

Laurentian University, Sudbury,

Ontario P3E 2C6, Canada

\footnotetext{
1. Carus-Wilson, C. Nature 44, 322-323 (1891)

2. Thomas, E. R. \& Jones, J. M. Victorian Nat. 81, 218-224 (1964).

3. Brown, A. E., Campbell, W. A., Jones, J. M. \& Thomas, E. R. Proc. Univ. Newcastle-upon-Tyne Phil. Soc, 1 (1), 1-21 (1964).

4. Campbell, W. A., Jones, J. M. \& Thomas, E. R. Proc. Univ. Newcastle-upon-Tyne Phil. Soc. 1 (14), 170-177 (1968).

5. Lindsay, J. F., Criswell, D. R., Criswell, T. L. \& Criswell, B. S. Geol Soc. Am. Bull. 87, 463-473 (1976).

6. Leach, M. F. \& Rubin, G. J. Acoust. Emiss. 11, 19-20 (1993).

7. Leach, M. E., Goldsack, D. E. \& Chartrand, H. J. Proc. 8th Pacif. Conf. NDT 55-62 (Taipei, Taiwan, 1995).

8. Leach, M. F., Goldsack, D. E. \& Kilkenny, C. Proc. 6th Conf. Acoust, Microseismic Activ. Geol. Struct. (Pennsylvania State Univ. Press, in the press).

9. Kronenberg, A. in Reviews in Mineralogy Vol. 29 (eds Meaney, P. H., Prewitt, C. T \& Gibbs, G. V.) Ch. 4 (Mineralogy Soc. Am., Washington $\mathrm{DC}, 1994$ ).

10. Henderson, P. Inorganic Geochemistry 256-257 (Pergamon, New
} York, 1982).

\section{Human haemoglobin}

\section{from transgenic tobacco}

Haemoglobin-based blood substitutes are currently being developed ${ }^{1,2}$ using haemoglobin from sources including outdated human or bovine blood, and expression in bacteria, yeasts or transgenic animals ${ }^{2}$. The oxygen affinity and stabilization of the purified haemoglobins can be controlled by a combination of crosslinking and sitedirected mutagenesis ${ }^{3,4}$, but other problems, such as haem oxidation and the presence of infectious agents, remain to be solved. Transgenic plants may be a suitable alternative source of haemoglobin as they constitute an inexpensive source of biomass, and transgenic crops are becoming commercially available ${ }^{5}$. Here we demonstrate the potential of this approach by co-expressing the $\alpha$ - and $\beta$-globins of the human haemo- globin $\mathrm{HbA}$ in transgenic tobacco plants, leading to the production of functional tetrameric haemoglobin.

We fused coding sequences of $\alpha$ - and $\beta$ globins to the sequence of the chloroplastic transit peptide (containing the first 57 residues of the protein precursor) of the small subunit of Rubisco from Pisum sativum L. (ref. 6). Co-expression was obtained with the binary plasmid pBIOC59 expressing fused $\alpha$ and $\beta$ sequences. This

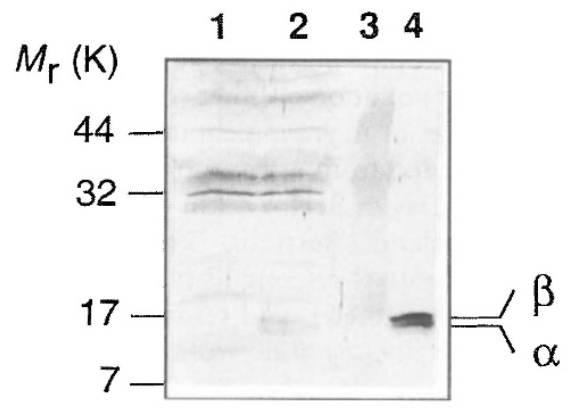

plasmid is derived from pGA492 (ref. 7) into which two expression cassettes, 'Pd35S-T35S' derived from pIIT60 (ref. 8), were inserted. Tobacco plants (Nicotiana tabacum var. Xanthi) were transformed using Agrobacterium tumefaciens strain LB4404 (ref. 9). We screened different plant-organ extracts from regenerated transformants for the presence of $\alpha$ - and $\beta$ globin chains by western blotting ${ }^{10}$.

Globin chains were detected in extracts

Figure 1 Western blot detection of the $\alpha$ and $\beta$-globins in crude seed extracts from transgenic tobacco plants. Lane $1,75 \mu \mathrm{g}$ wildtype extracted plant protein. Lane 2, $75 \mu \mathrm{g}$ transgenic extracted plant protein. Lane 3 , molecular mass markers. Lane 4, $50 \mathrm{ng}$ purified human HbA. The western blot analysis was done with rabbit anti-human haemoglobin immunoserum and goat antirabbit IgG antibody coupled to alkaline phosphatase. 